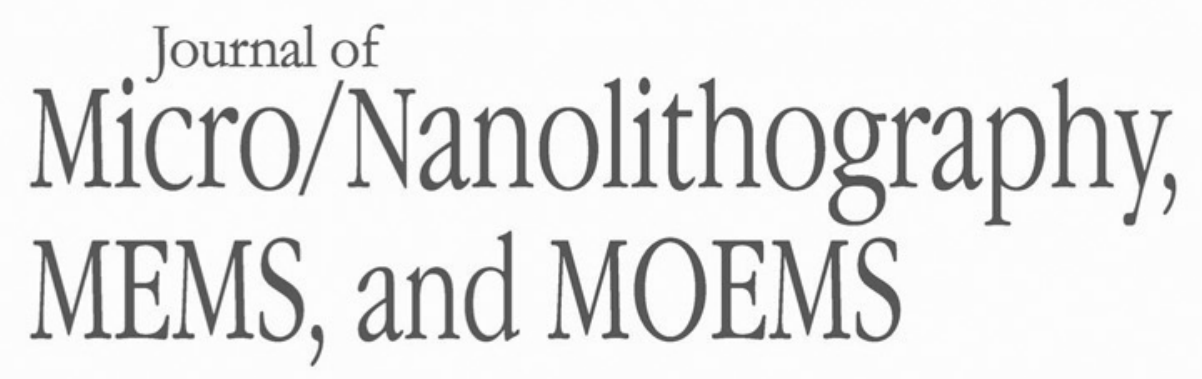

Nanolithography.SPIEDigitalLibrary.org

\title{
2016 List of Reviewers
} SPIE. “2016 List of Reviewers,” J. Micro/Nanolith. MEMS MOEMS 16(1), 010102 (2017), 
The Journal of Micro/Nanolithography, MEMS, and MOEMS would like to sincerely thank the following individuals who served as reviewers in 2016 . The success of our publication hinges on the voluntary contributions of time and energy put forth by these professionals.

Frank Abboud

Tsukasa Abe

Hillar Aben

Md. Shamim Ahsan

Seyed Allameh

James Allen

Gilles Amblard

Masafumi Asano

Mohamed Asbahi

Mark Auslender

Christopher Ausschnitt

Mohamed Bahnas

Sarfaraz Ali Baig

Artur Balasinski

Xinyu Bao

Bryan Barnes

Eytan Barouch

Ralf Bauer

Chris Bencher

Markus Benk

Karl Berggren

Ali Asgar Bhagat

Alexei Bogdanov

Ingo Bork

Anatoly Bourov

Robert Brainard

Alan Brodie

Robert Bruce

Timothy Brunner

Benjamin Bunday

Martin Burkhardt

Neal Butler

Ziliang Cai

Giuseppe Calafiore

Sergio Calixto-Carrera

Sergio Camacho-Leon

James Cameron

Leanne Chan

Lingqian Chang

Robin Chao

Weilun Chao

Chun-Kuang Chen

Frederick Chen

Yijian Chen

Mark Ming-Cheng Cheng

Ping Cheng

Hai-Pang Chiang

Yi Chiu

Seungkeun Choi

Michael Chomat

Shuo-Yen Chou

Danut Adrian Cojoc

Matthew Colburn

Brid Connolly

Vassilios Constantoudis

William Cowan

Shawn Cunningham
Xuhan Dai

Benyamin Davaji

Wyatt Davis

Sukomal Dey

Yuzhe Ding

Dhairya Dixit

Gregory Doerk

Yasin Ekinci

Andreas Erdmann

Yongfa Fan

Jo Finders

Johann Foucher

John Fourkas

Emily Gallagher

He Gao

A. Gerardino

Farshid Ghasemi

Sertan Gokce

Yuri Granik

Jeffrey Gregory

Timothy Groves

Tetsuo Harada

Faezeh Arab Hassani

Benjamin Hatton

Keiichiro Hitomi

Chong Pei Ho

Stephanie Hoeppener

Ulrich Hofmann

Ehsan Hosseiniyan

Matiar Howlader

Su-mi Hur

Paul Hurley

Phillip Hustad

Soichi Inoue

Mikkel Jorgensen

II Woong Jung

Larissa Juschkin

Young Bok (Abraham) Kang

Karim Karim

Bryan Kasprowicz

Rafal Kasztelanic

Daman Khaira

Seok-min Kim

SeongSue Kim

Tae-Gon Kim

Kurt Kimmel

Edward Kinzel

Joachim Knittel

Kahhow Koh

Pieter Kruit

Yi-sha Ku

Aleksandr Kuchmizhak

Maria Kufner

Kafai Lai

Barton Lane

Bertrand Le-Gratiet

Byoung-Ho Lee
Chengkuo Lee

Eon Soo Lee

Philippe Leray

Harry Levinson

Cheng-Syun Li

Fengyuan Li

Han Li

Lifeng $\mathrm{Li}$

Lijie Li

Ming-Huang Li

Wei-Chang Li

Ted Liang

Lars Liebmann

Dmitriy Likhachev

Chenxi Lin

Chih-Ming Lin

Vincent Lin

Anna Lio

Patrick Langechuan Liu

Chiung-Cheng Lo

Daniel Lopez

Gian Lorusso

Liang Lou

Tom Lucatoro

Xiangang Luo

Yuansheng $\mathrm{Ma}$

Chris Mack

John Maltabes

Geminiano Martinez-Ponce

Shinji Matsui

Robert Meeks

Sohan Mehta

Lawrence Melvin III

Ichiko Misumi

Joydeep Mitra

Ryan Miyakawa

Masato Mizukami

Mohammad Moghimi

Ali-Reza Moradi

Katrina Morgan

Yasutaka Morikawa

Alain Moussa

Muhammad Mujeeb-U-Rahman

Jan Mulkens

Viviana Mulloni

Lawrence Muray

Alex Murokh

Seiji Nagahara

Wataru Nakagawa

Norio Nakamura

George Nehmetallah

Mark Neisser

Arashk Norouzpour-Shirazi

Jonghyun Oh

Kazuhiko Omote

Katsura Otaki

Melih Ozlem 
Muthukumaran Packirisamy

David Pan

Linyong Pang

Eric Panning

Dongwoon Park

Danping Peng

Jan Hendrik Peters

Vicky Philipsen

Wibool Piyawattanametha

Amyn Poonawala

Michael Postek

Pushpa Pudasaini

Lei Qiao

Jed Rankin

Benjamen Rathsack

Christopher Raymond

Xiang Ren

Stephen Renwick

Douglas Resnick

Paulina Rincon Delgadillo

Gijsbert Rispens

Klaus-Dieter Roeth

Lucia Romano

Alan Rosenbluth

Shimul Saha

Ildar Salakhutdinov

Thilo Sandner

Iqbal Saraf

Jochen Schacht

Franklin Schellenberg

Kristin Schmidt
Tamar Segal-Peretz

Matthew Sendelbach

Apo Sezginer

Peng Shao

Akshdeep Sharma

Wen-Pin Shih

Tsutomu Shoki

Sameet Shriyan

Mark Somervell

Amelia Carolina Sparavigna

David Stegall

Dan Stieler

Hyo Seon Suh

Chih-Ming Sun

Jyh-Rou Sze

Niall Tait

Kiwamu Takehisa

Amir Tavakkoli

Tsuneo Terasawa

James Thackeray

Ye Tian

Kagan Topalli

Juan Torres

Ilhami Torunoglu

Julius Tsai

Sheng-Hsiang Tseng

Chingfu Tsou

Jacek Tyminski

Mitsuru Ueda

Alessandro Vaglio Pret

Jan van Schoot
Nilesh Vasa

Dmitry Vengertsev

Marc Verschuuren

Dung-An Wang

Lon Wang

Nan Wang

Wentao Wang

Masahiro Watanabe

Takeo Watanabe

Francois Weisbuch

Matthew Wormington

Lingqi Wu

Wen Wu

Qiangfei Xia

Xiao Xiang

Shuaigang Xiao

Alexander Yakunin

Bin Yang

Hiroshi Yasuda

Hiroshi Yoshida

Mohammad Younis

Bei Yu

Shinn-Sheng Yu

Frederic Zamkotsian

Kai Zhang

Songsong Zhang

Yuebing Zheng

Mingyu Zhong

Fan Zhou

Huchuan Zhou

Haoshen Zhu 